\title{
Targeted genome reconstruction strategy for endosymbionts in eukaryotic genomes
}

\author{
Surya Saha ${ }^{1 *}$, Wayne B Hunter ${ }^{2}$, Magdalen Lindeberg ${ }^{1}$ \\ From Beyond the Genome 2012 \\ Boston, MA, USA. 27-29 September 2012
}

\begin{abstract}
Background
The human microbiome project [1] is the first systematic and large-scale survey of microbial communities present in a eukaryotic metagenome. The availability of low-cost high-throughput next-generation sequencing technologies that enable us to sequence eukaryotes to a high depth of coverage presents an opportunity for in silico discovery of endosymbionts. We describe a method for mining a whole genome shotgun metagenome from an insect to identify members of the endosymbiont community, followed by reconstruction and validation of a highquality draft microbial genome. The Asian citrus psyllid (Diaphorina citri Kuwayama or ACP) is host to 7+ bacterial endosymbionts [2] and is the insect vector of Candidatus Liberibacter asiaticus (Las), which is the causal agent of citrus greening, a disease costing the Florida citrus industry US $\$ 3.63$ billion and 6,611 jobs since 2006 . Citrus greening is a complex patho-system that involves interactions between the psyllid vector, the citrus host, and Candidatus Liberibacter asiaticus.
\end{abstract}

\section{Materials and methods}

To gain a better understanding of the ACP endosymbiont community, DNA from ACP was sequenced to 108X coverage to produce paired-end and mate-pair libraries. Initial analyses focused on Wolbachia, an alpha-proteobacterial primary endosymbiont typically found in the reproductive tissues of ACP and other arthropods. The metagenomic sequences were mined for Wolbachia (wACP) reads using four sequenced Wolbachia genomes as bait. Putative wACP reads were then assembled using Velvet and MIRA3 assemblers. The resulting wACP contigs were annotated using the RAST pipeline and compared with the closest sequenced Wolbachia from an insect genome:

'Department of Plant Pathology and Plant-Microbe Biology, Cornell University, Ithaca, NY, USA

Full list of author information is available at the end of the article
Wolbachia endosymbiont of Culex quinquefasciatus (wPip). MIRA3 was able to reconstruct a majority of the wPip coding sequence regions and was therefore selected for scaffolding using large insert mate-pair libraries. The wACP scaffolds were further improved using Abacas and Mauve contig mover with wPip as reference genome to orient and order the contigs.

\section{Results and conclusions}

We validated the final wACP scaffold by comparing all wACP proteins against the four sequenced Wolbachia genomes. We ran OrthoMCL and selected core and shared Wolbachia proteins where the classification was highly conserved $(>80 \%)$ across all runs. In order to determine the presence of the core Wolbachia proteins in our wACP scaffold, we compared wACP proteins with labeled Wolbachia proteins. 1,164/1,213 wACP proteins had matches, of which 669 were to core proteins. This number compares favorably to the number of core proteins (670) found in sequenced Wolbachias. The scope of endosymbiont characterization was expanded beyond wACP using 16S rDNA and partial 23S rDNA analysis [2] as a guide. Reads from the ACP metagenome were mapped to reference genomes of candidate endosymbionts and mapped regions were analyzed manually. We are currently screening out rRNA regions and using taxonomic classification in order to determine the specificity of these regions to the genome. Results will be presented regarding targeted assembly methods, validation metrics and effectiveness of taxonomic classification strategies for metagenomics.

\section{Author details}

${ }^{1}$ Department of Plant Pathology and Plant-Microbe Biology, Cornell University, Ithaca, NY, USA. ${ }^{2}$ USDA-ARS, US Horticultural Research Laboratory, Fort Peirce, FL, USA.

Published: 1 October 2012 


\section{References}

1. Human Microbiome Project Consortium: A framework for human microbiome research. Nature 2012, 486:215-221.

2. Marutani-Hert M, Hunter WB, Morgan JK: Associated bacteria of Asian citrus psyllid (Hemiptera: Psyllidae: Diaphorina citri). Southwestern Entomologist 2011, 36:323-330.

doi:10.1186/1753-6561-6-S6-P33

Cite this article as: Saha et al:: Targeted genome reconstruction strategy for endosymbionts in eukaryotic genomes. BMC Proceedings 2012 6(Suppl 6):P33.

Submit your next manuscript to BioMed Central and take full advantage of:

- Convenient online submission

- Thorough peer review

- No space constraints or color figure charges

- Immediate publication on acceptance

- Inclusion in PubMed, CAS, Scopus and Google Scholar

- Research which is freely available for redistribution

Submit your manuscript at www.biomedcentral.com/submit
Ciomed Central 